\title{
DNA dC to dU-Editing Enzyme APOBEC-3B
}

National Cancer Institute

\section{Source}

National Cancer Institute. DNA dC to dU-Editing Enzyme APOBEC-3B. NCI Thesaurus.

Code C117003.

DNA dC->dU-editing enzyme APOBEC-3B (382 aa, $\sim 46 \mathrm{kDa}$ ) is encoded by the human APOBEC3B gene. This protein plays a role in the inhibition of replication of viral genomes. 